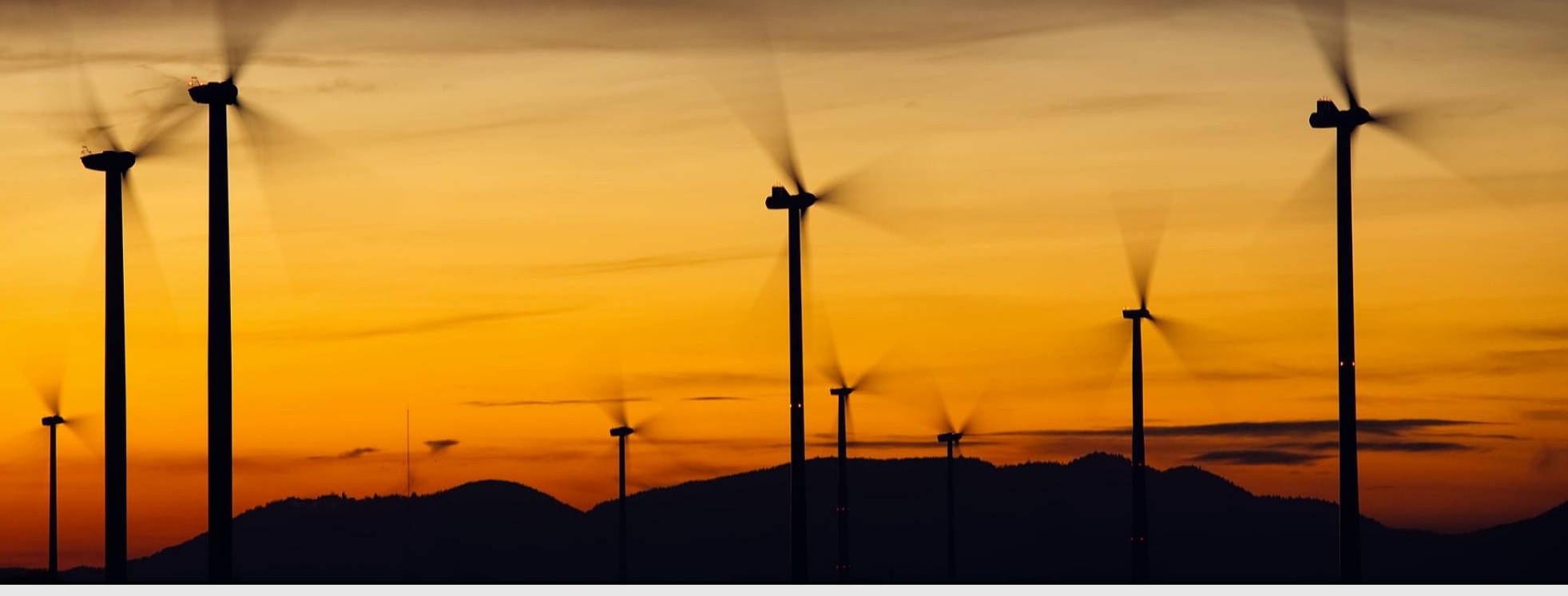

\title{
Predicción por conjuntos y estimaciones de incerti- dumbre en predicción de energía eólica
}

\author{
Alejandro Catalina y José R. Dorronsoro \\ Departamento de Ingeniería Informática e Instituto de Ingeniería del Conocimiento, UAM
}

Los Anemoi eran dioses del viento, se correspondían con los puntos cardinales y que estaban relacionados con las distintas estaciones y estados meteorológicos. Los cuatro Anemoi eran: Bóreas (norte - invierno), Noto (sur - verano), Euro (este - otoño) y Céfiro (oeste - primavera). Los Anemoi eran hijos de Astreo (también conocido como padre de las estrellas) y de Eos, diosa del amanecer. Otros hijos importantes de esta pareja fueron los cinco Astra Planeta (estrellas errantes, es decir, planetas): Fenonte (Júpiter), Faetonte (Saturno), Piroente (Marte), Fósforo (Venus) y Estilbo (Mercurio).

https: //arescronida. wordpress.com/tag/anemoi/ - ARES CRONIDA

Por razones tanto ecológicas como económicas se está produciendo un rápido crecimiento de las energías renovables y, en particular, de la energía eólica. Los sistemas de predicción por conjuntos (SPC) basados en modelos meteorológicos numéricos pueden ser una herramienta muy útil tanto para mejorar la calidad de las predicciones de energía renovable como para proporcionar estimaciones útiles de la incertidumbre asociada a dichas predicciones. En este capítulo se considerará la aplicación a tal efecto de los SPC meteorológicos proporcionados por el European Centre for Medium-range Weather Forecasts (ECMWF), tanto a nivel de la predicción local de energía eólica en un solo parque, como en el área mucho más amplia de la España peninsular mostrando que, si bien las predicciones deterministas pueden tener alguna ventaja sobre las de los ensembles en términos de resolución espacial, los SPC pueden usarse para derivar intervalos de incertidumbre satisfactorios. Fuera del alcance de este capítulo queda un estudio sobre la utilización de la PDF que proporcionan los SPC para ofrecer predicciones puramente probabilistas.

Palabras clave: sistemas de predicción por conjuntos aplicados a la energía eólica, predicción probabilista para la previsión de energía eólica, modelos neuronales y SVR. 


\subsection{Introducción}

En parte por consideraciones sobre el cambio climático y en parte y cada vez más por razones económicas, se está produciendo un rápido crecimiento de las energías renovables y, en particular, de la energía eólica. Como consecuencia, una predicción precisa de dicha energía es clave para su gestión e integración en los distintos sistemas eléctricos. Hay dos objetivos primordiales para dicha predicción. Por un lado, hacer que las mismas tengan una precisión tan alta como sea posible. Por otro lado, y dada variabilidad meteorológica, acompañar dichas predicciones con intervalos de incertidumbre fiables.

Para horizontes de uno a varios días la predicción meteorológica numérica o Numerical Weather Prediction (NWP, 10 en la página 129) es la entrada habitual a la hora de aplicar modelos de aprendizaje automático (AA) y entre las NWP más establecidas están sin duda la del European Centre for Medium-Range Weather Forecasts (ECMWF, cap. 19 en la página 289). Su modelo determinista, ECHRES (sec. 19.2 en la página 291) está basado a grandes rasgos en la mejor estimación de los parámetros del modelo y de sus condiciones iniciales. Este modelo se integra con las resoluciones más finas disponibles (actualmente $9 \mathrm{~km}$ de resolución espectral, disponible en rejilla a $0,1^{\circ}$ $\mathrm{y}$, en los periodos de los experimentos realizados, 16 $\mathrm{km}$ de resolución espectral, disponible en rejilla a $\left.0,125^{\circ}\right)$. No obstante, las predicciones meteorológicas están sujetas a incertidumbre y por ello los modelos numéricos globales desarrollan la denominada predicción probabilista de conjuntos o ensembles [2,7]. En el caso del ECMWF, esta predicción probabilista (ECENS, sec. 19.3 en la página 293) está formada por un modelo de control, esencialmente coincidente con el determinista pero con una menor resolución de integración (actualmente $18 \mathrm{~km}$ de resolución espectral, disponible en rejilla a $0,2^{\circ}$ y, en los periodos de los experimentos realizados, $32 \mathrm{~km}$ de resolución espectral, disponible en rejilla a $0,25^{\circ}$ ). Hay además 50 predicciones adicionales que toman como condiciones iniciales unas ciertas perturbaciones de las de control.

Es natural preguntarse cómo aprovechar los ensembles para complementar las predicciones obtenidas con el modelo determinista. Una primera intuición sugiere pensar que a horizontes cortos las predicciones de energía obtenidas mediante ensembles NWP deberían tener unos errores comparables a los de las predicciones de energía obtenidas mediante el modelo determinista (aunque tal vez ligeramente superiores), mientras que a horizontes más lejanos su mayor información sí sería de mayor utilidad. Por otro lado, los ensembles deberían poder utilizarse para proporcionar intervalos de incertidumbre a la predicción de energía eólica.

En este capítulo, donde resumimos brevemente un trabajo más amplio [3] trataremos ambas cuestiones, considerando la predicción de energía eólica tanto para toda la península ibérica como para un único parque, Sotavento, situado en Galicia. Como modelos de aprendizaje automático (AA) emplearemos redes neuronales $(\mathrm{NN})$ y máquinas de vectores de soporte para regresión (SVR). En esta nota, que tiene sobre todo un carácter de primer análisis de lo que puede conseguirse con los ensembles, consideraremos dos horizontes de predicción, para el mismo día en que se generan las predicciones y a dos días.

\subsection{Modelos Neuronales y SVR}

En nuestros experimentos trabajaremos con redes neuronales sencillas y con máquinas de vectores de soporte para regresión, que describimos a continuación.

Para una muestra de tamaño $N\left\{\left(x^{1}, y^{1}\right), \ldots,\left(x^{N}, y^{N}\right)\right\}$ una red neuronal [1] trata de minimizar la siguiente función de pérdida regularizada $\left(L_{2}\right)$

$$
e_{N N}(w)=\frac{1}{2 N} \sum_{p}\left(y^{p}-f\left(x^{p}, w\right)\right)^{2}+\frac{\alpha}{2}\|w\|_{2}^{2}
$$

donde $f(x, w)$ denota la salida para $x$ de una red neuronal cuya arquitectura determina un conjunto de pe$\operatorname{sos} w$. Trabajaremos con la implementación en scikitlearn [6] de redes fully connected con el mismo número de neuronas por capa, concretamente 2 capas con 100 neuronas cada una. Hemos fijado la función de activación como ReLUs y los parámetros por defecto para el solver adam. Por tanto sólo hiperparametrizaremos $\alpha$, la constante de penalización de la regularización. Por otro lado, la función de pérdida para las máquinas de vectores soporte para regresión (SVR) [1] es la siguiente

$$
e_{S V R}=\sum_{p}\left[y^{p}-w \cdot x^{p}-w_{0}\right]_{\varepsilon}+\frac{1}{2 C}\|w\|_{2}^{2},
$$

donde de nuevo usamos regularización $L_{2}$ y la pérdida $\varepsilon$-insensitive. 
Generalmente esta función de pérdida se reescribe como un problema con restricciones que posteriormente se transforma mediante la teoría lagrangiana en un problema dual mucho más sencillo, que es el que finalmente se resuelve. El problema dual solo involucra patrones a través del producto escalar, lo que permite el conocido truco del núcleo (kernel trick), que reemplaza los productos escalares $x \cdot x^{\prime}$ con los valores $k\left(x, x^{\prime}\right)$ de un núcleo definido positivo. La opción habitual es usar el núcleo gaussiano, que resulta en un modelo SVR con tres hiper-parámetros, el coeficiente de regularización $C$, el valor de $\varepsilon$ de la función de pérdida y la anchura del núcleo $\gamma$. Aquí también usaremos la implementación en scikit-learn para SVR, así como su módulo para búsqueda en rejilla de hiperparámetros, donde utilizaremos datos del año 2013 como entrenamiento y de 2014 como validación; los datos de 2015 serán para test.

\subsection{Experimentos}

Entrenaremos los modelos determinista y control emparejando predicciones NWP para el mismo día con los correspondientes valores horarios de energía. Las producciones de Sotavento están accesibles en su web mientras que las de la España peninsular han sido proporcionadas por Red Eléctrica de España (REE). Las predicciones ensemble de energía corresponden a la aplicación de los modelos NN o SVR entrenados sobre el modelo NWP control a las 50 predicciones NWP del ensemble.

\subsubsection{Predicciones Deterministas vs En- sembles}

El primer experimento será una comparación entre la precisión de los modelos NN y SVR aplicados a la NWP determinista y de control, así como la media y mediana de las predicciones obtenidas sobre los 50 ensembles NWP. En todos los casos usaremos como variables de predicción las siguientes:

1. Componentes $\mathrm{U}$ y V de la velocidad del viento a 10 y 100 metros así como sus módulos.

2. Presión en superficie.

3. Temperatura a 2 metros.

4. Conversión del viento a 10 y 100 metros a potencia usando una curva genérica que satura a $20 \mathrm{~m} / \mathrm{s}$.

\begin{tabular}{llcccc}
\hline & \multicolumn{2}{c}{ Sotavento } & \multicolumn{2}{c}{ REE } \\
& MAE & RMSE & MAE & RMSE \\
\hline \multirow{4}{*}{ MLP } & Det & 5.86 & 8.50 & 2.76 & 3.66 \\
& Control & 6.84 & 9.12 & 2.68 & 3.67 \\
& Mean & 6.01 & 8.78 & 2.68 & 3.65 \\
& Median & 5.99 & 8.78 & 2.68 & 3.64 \\
& Det (2d) & 7.25 & 10.50 & 4.60 & 5.77 \\
& Mean (2d) & 8.37 & 11.35 & 6.17 & 7.55 \\
& Median (2d) & 8.21 & 11.34 & 6.14 & 7.54 \\
\hline \multirow{4}{*}{ SVR } & Det & 5.80 & 8.52 & 2.54 & 3.37 \\
& Control & 6.34 & 9.13 & 2.56 & 3.40 \\
& Mean & 5.85 & 8.72 & 2.56 & 3.38 \\
& Median & 5.84 & 8.74 & 2.56 & 3.39 \\
& Det (2d) & 7.19 & 10.57 & 4.63 & 5.80 \\
& Mean (2d) & 9.31 & 12.20 & 6.70 & 8.14 \\
& Median (2d) & 9.23 & 12.22 & 6.66 & 8.10 \\
\hline
\end{tabular}

Tabla 39.1: MAE y RMSE para los modelos NN y SVR en el mismo día y para dos días hacia adelante. 
Estas variables se normalizan de forma adecuada y la energía se considera a una escala 0-100. Utilizaremos una resolución de $0,25^{\circ}$ en Sotavento, puesto que es la única disponible en los datos de conjuntos. Por razones de complejidad computacional, usaremos para la península una resolución menor de $0,5^{\circ}$. Esto resulta en 1200 variables en Sotavento y 5520 en la península.

En la Tabla 39.1 mostramos los errores MAE y RMSE (sec. 15.2.3 en la página 211) de los diferentes escenarios. Como puede verse, el mejor modelo es SVR, claramente a dos días y también, aunque menos, para el mismo día. Destaca también que en el mismo día la mediana de los conjuntos es el segundo mejor modelo, seguido de cerca de la media, mientras el el modelo de control está claramente detrás. En cualquier caso, si bien en el mismo día medias y medianas están cerca del modelo determinista, a 2 días se quedan bastante atrás. Pero hay que observar que se trata de un primer acercamiento a la utilidad potencial de los ensembles, en el que se utilizan por separado el miembro de control, la mediana o la media del ensemble y se comparan con el determinista. Una posibilidad para futuros trabajos es utilizar la PDF completa.

\subsubsection{Intervalos de Incertidumbre}

Otro uso posible de las predicciones ensemble es derivar intervalos de confianza para las predicciones de energía. Sin embargo, la opción natural de calcularlos directamente en función de la dispersión de las predicciones ensemble no es aplicable, pues las predicciones
NWP de los ensembles pueden ser subdispersivas [4, 5] (aunque esta cuestión requiere un estudio posterior, ver también sec. 13.6.5 en la página 181 y 15.8.2 en la página 223).

Por ello aplicaremos a dichas dispersiones una calibración relativamente sencilla, en concreto

$$
\hat{p}_{d e t}^{h} \pm \delta \frac{m_{d e t}}{m_{\text {ens }}} s p r_{\text {ens }}^{h}(s),
$$

donde $\hat{p}_{\text {det }}^{h}$ es la predicción determinista para la hora $h, \delta$ es un parámetro de calibración a estimar, $m_{d e t}$ es la mediana de los errores deterministas, $m_{\text {ens }}$ es la mediana de la dispersión de los conjuntos y $\operatorname{spr}_{e n s}^{h}(s)$ es la diferencia entre los percentiles $\frac{s}{2}$ y $100-\frac{s}{2}$ de las predicciones ensemble para la hora $h$, siendo $s$ el nivel de confianza deseado. El parámetro $\delta$ se estima por validación cruzada para lo que usamos como función de pérdida el porcentaje de producciones fuera del intervalo $I_{\delta, s}$ definido por la ecuación (39.3)

$$
\operatorname{per}_{\text {err }}(\delta, s)=\mid\left\{\% \text { of test residuals } \in I_{\delta, s}\right\}-(100-s) \mid \text {. }
$$

En la Tabla 39.2 mostramos los resultados para intervalos del 50 y $90 \%$; como puede verse, el nivel de incertidumbre es muy cercano a la confianza deseada. También destaca que en este experimento no hay una diferencia significativa entre la red neuronal y el modelo SVR, probablemente porque el proceso de optimización de $\delta$ se adapta a los errores de los respectivos modelos. Igualmente, tampoco hay una gran diferencia entre la calidad de los intervalos del mismo día y a 2 días.

\begin{tabular}{lccccc}
\hline & & \multicolumn{2}{c}{ Sotavento } & \multicolumn{2}{c}{ REE } \\
& Confianza & $0 \mathrm{~d}$ & $2 \mathrm{~d}$ & $0 \mathrm{~d}$ & $2 \mathrm{~d}$ \\
\hline \multirow{2}{*}{ MLP } & 50 & 49.70 & 50.01 & 53.42 & 47.07 \\
& 90 & 90.55 & 90.22 & 92.00 & 89.98 \\
\hline \multirow{2}{*}{ SVR } & 50 & 51.27 & 48.71 & 50.73 & 47.84 \\
& 90 & 89.31 & 89.71 & 90.73 & 91.93 \\
\hline
\end{tabular}

Tabla 39.2: Porcentajes de incertidumbre deseados (segunda columna) y porcentajes reales de las predicciones dentro de cada intervalo de incertidumbre para el mismo día y para dos días hacia adelante. 


\subsection{Discusión y Conclusiones}

La principal conclusión de las secciones anteriores es que los ensembles NWP pueden enriquecer la predicción de energía eólica y proporcionar intervalos de incertidumbre fiables. Pero, por otro lado, hay que tener en cuenta que la generación de estos datos supone un coste computacional sustancialmente mayor, tanto a la hora de emitir y manejar predicciones como en almacenamiento. En cualquier caso, puede haber otras ventajas derivadas del uso de los ensembles. En este ejercicio se ha utilizado el miembro de control, la mediana o la media del ensemble como predicciones separadas, pero no la PDF en su conjunto como predicción propiamente probabilista. Tampoco se han considerado horizontes de predicción más allá de 1-2 días. Además, una forma de mejorar las predicciones puede ser considerando la posible relación entre la inestabilidad de la atmósfera y la dispersión de los conjuntos. Cuando la inestabilidad sea pequeña podría esperarse una dispersión pequeña, y viceversa en el caso contrario. Todas estas son cuestiones interesantes que merecerían un estudio específico.

Agradecimientos. Con soporte parcial de los proyectos TIN2013-42351-P, TIN2016-76406-P y S2013/ICE-2845 CASI-CAM-CM, así como del proyecto FACIL-Ayudas Fundación BBVA a Equipos de Investigación Científica 2016 y de la Cátedra UAMADIC en Ciencia de Datos y Aprendizaje Automático. Agradecemos a Red Eléctrica de España los datos de producción eólica en España y el uso de las instalaciones del Centro de Computación Científica (CCC) en la UAM. 


\subsection{Referencias}

[1] BIshop, Christopher M. Pattern recognition and machine learning. springer, 2006. DOI: 10.1117/1.2819119 (citado en página 608).

[2] Callado, Alfons y col. "Ensemble Forecasting". En: Climate Change and Regional/Local Responses. Editado por RAY, Pallav. InTech, mayo de 2013. ISBN: 978-95351-1132-0. DOI: 10 . 5772/55699 (citado en página 608).

[3] Catalina, Alejandro y Dorronsoro, José R. "NWP Ensembles for Wind Energy Uncertainty Estimates". En: Workshop on Data Analytics for Renewable Energy Integration. Lecture Notes in Artificial Intelligence. Springer, Cham, sep. de 2017, páginas 121-132. DOI: 10 . 1007/978-3-31971643-5_11 (citado en página 608).
[4] GIEBEL, Gregor y col. Wind power prediction using ensembles. Informe técnico. 2005 (citado en página 610).

[5] Leutbecher, Martin y col. "Ensemble forecasting". En: Journal of Computational Physics 227.7 (2008), páginas 3515-3539. ISSN: 00219991. DOI: 10 . $1016 / \mathrm{J}$. JCP . 2007.02.014 (citado en página 610).

[6] Pedregosa, Fabian y col. "Scikit-learn: Machine learning in Python". En: Journal of Machine Learning Research 12.Oct (2011), páginas 2825-2830 (citado en página 608).

[7] WMO. Guidelines on Ensemble Prediction Systems and Forecasting. WMO. World Meteorological Organization (WMO), 2012. URL: http : // library . wmo . int/pmb_ ged/wmo_1091_en . pdf (citado en página 608). 
